\title{
A Case Study of a Female Ultramarathon Wheelchair Road User
}

\author{
Alfred F. Morris, Ph.D., F.A.C.S.M. \\ Armed Forces Staff College, 7800 Hampton Boulevard, Norfolk, \\ Virginia, VA 23511-6097, U.S.A.
}

\begin{abstract}
Summary
This unique report contains selected physical and structural characteristics of a female ultramarathon wheelchair road racer. When tested, this 25-year-old athlete weighed 65.6 kilograms, with a height of 171.4 centimetres. The resting heart rate was 57 beats per minute, which rose to a maximum of 161 during a progressive, arm-cranking, maximal work task. Maximum oxygen uptake was $21.0 \mathrm{ml} \times \mathrm{kg}^{-1}$ $\times$ min $^{-1}$. These values are significant, considering that this individual had traumatic paraplegia at the T-3/T-4 spinal level. She also had slight weakness of the upper extremities. It is suggested that with a graduated and intelligently structured training programme, greatly enhanced endurance capacity can be achieved. With the type of training outlined in this paper, an athlete who performs in a wheelchair can be conditioned to race successfully over distances of 26 to 50 miles.
\end{abstract}

Key words: Wheelchair female athlete; Aerobic exercise; Human endurance performance

\section{Introduction}

Human performance in endurance sport activities often takes many continuous minutes, even hours, of sustained, hard effort. Any single extreme performance may therefore serve as an accurate measurement of the endurance fitness capacity of an individual athlete. For an athlete in a long distance race to complete a marathon $(42 \cdot 2 \mathrm{Km})$ or ultramarathon event, and to finish in a fast time, indicates an extraordinary level of endurance fitness in that athlete. Ultramarathon events in road racing consist of events of $50 \mathrm{Km}$ or more.

In the laboratory, measurement of maximal aerobic fitness $\left(\dot{\mathrm{V}}_{\mathrm{O}_{2}} \max \right)$ and sustained, near maximal heart rates have been shown to be excellent indicators of endurance of cardiorespiratory fitness (Mahler and Loke, 1985). In ablebodied athlete values of 60,70 , and even in excess of $80 \mathrm{ml} \times \mathrm{kg}^{-1} \times \mathrm{min}^{-1}$ have been reported (Åstrand and Rodahl, 1977; Åstrand and Saltin, 1961). It has been reported that maximal oxygen uptake is related to the amount of active 
muscle tested in the work task (Bergh, Kanstrup, and Ekblom, 1976; Secher, Ruberg-Larsen, Binkhorst and Bonde-Petersen, 1974). Also, Morris (1982, 1983, $1984,1985)$ has indicated that performance in a wheelchair task is related to active musculature innervated and controlled by the wheelchair competitor.

There is little scientific information on endurance sport performance in wheelchair athletes. There are no data on female athletes who are disabled, but who compete in ultramarathon road racing events. Therefore, the purpose of this study was to determine the cardiorespiratory performance and endurance capacity in a young woman athlete who competes in ultramarathon events in a wheelchair. This exceptionally gifted athlete was in condition to race a 50-mile ultramarathon road race.

\section{Methods}

\section{Subject}

The subject for this case study, who signed Informed Consent forms before being evaluated in a laboratory, was a 25-year-old female athlete who had sustained an accident while pot-holing (spelunking). As a result of this injury, the subject was rendered paraplegic at the T3/4 spinal level. There was minimal residual paralysis of the upper extremities. The accident occurred when the athlete was in high school.

This person had trained for an ultramarathon road race competition in Chicago, sponsored by the American Medical Joggers Association. She finished a 50-mile race in a time of 6 hours and 54 minutes. To our knowledge, this performance ranks as a world best for a woman athlete competing in a wheelchair.

She was a veteran of marathon road racing events, completing Boston and Chicago marathons with times under 3 hours and 30 minutes, and had trained, averaging nearly 50 miles per week, for several years prior to this attempt at an ultramarathon distance of 50 miles. This amount and kind of training qualified her as an endurance athlete (Morris, 1984).

\section{Measurement of body density}

Body density $\left(D_{b}\right)$ was measured by the hydrostatic technique of underwater weighing, using the procedures and instrumentation suggested by Akers and Buskirk (1969). Underwater weight was measured in a weighing platform resting on force transducers (Datronic Corporation, Model 152A-25; Hewlett-Packard, Model 7123A). At the end of a forced expiration, residual volume measurements were made. The subject was made familiar with the underwater weighing by several practice trials. This was followed by three separate weight determinations with the average value derived from three scores used as the actual score.

\section{Cardiorespiratory testing}

Measurements of cardiorespiratory function were assessed during a progressive, arm-cranking, maximal endurance work task. A Monark cycle ergometer (Model 881) was converted to arm-cranking by appropriate bicycle chain and pedal 
instrumentation. The arm ergometry protocol involved six steps. There was an initial pre-warm-up period, followed by the six steps, lasting one minute each, raising the increment of resistance on the arm ergometer from 144 to $528 \mathrm{Kpm} / \mathrm{min}$. The arm-crank rate was kept at 50 revolutions per minute. Being a well trained endurance athlete, the subject was asked to work until exhaustion.

\section{Results}

The athlete weighed 65.5 kilograms, and had a measured height of 171.4 centimetres (in the supine position). The resting heart rate was 57 beats per minute (in the supine position), and a maximum heart rate of 161 beats per minute was achieved during the progressive, arm-cranking, maximum endurance work task. The arm ergometry protocol, as well as cardiorespiratory results, are given in Table 1.

Table Arm ergometry protocol and cardiorespiratory results

\begin{tabular}{lccccccc}
\hline Parameters & Min & 2 & 3 & 4 & 5 & 6 & 7 \\
& $\mathrm{Kg}$ & $0 \cdot 5$ & $1 \cdot 0$ & $1 \cdot 0$ & $1 \cdot 5$ & $1 \cdot 5$ & $2 \cdot 0$ \\
& Crank Rate & 48 & 48 & 48 & 50 & 50 & 44 \\
& $\mathrm{Kpm}$ & 144 & 288 & 288 & 450 & 450 & 528 \\
\hline$\dot{\mathrm{V}}_{\mathrm{E}}(1 / \mathrm{min})$ & & $22 \cdot 8$ & $28 \cdot 2$ & $36 \cdot 0$ & $50 \cdot 4$ & $51 \cdot 2$ & $55 \cdot 2$ \\
$\dot{\mathrm{V}}_{\mathrm{O}_{2}}(1 / \mathrm{min})$ & & 0.95 & 1.05 & 1.25 & 1.37 & 1.37 & 1.32 \\
$\dot{\mathrm{V}}_{\mathrm{O}_{2}}(\mathrm{ml} / \mathrm{min} / \mathrm{Kg})$ & & 14.6 & 16.2 & 19.2 & 21.1 & 21.1 & 20.3 \\
$\dot{\mathrm{V}}_{\mathrm{CO}}(1 / \mathrm{min})$ & & 0.74 & 0.92 & 1.21 & 1.42 & 1.41 & 1.36 \\
Heart Rate & & 119 & 137 & 147 & 154 & 154 & 161 \\
$\quad$ (beats per min) & & & & & & & \\
\hline
\end{tabular}

A maximum $\dot{\mathrm{V}}_{\mathrm{O}_{2}}$ (STPD) value achieved was 1.37 litres per minute. $\dot{\mathrm{V}}_{\mathrm{O}_{2}}$ max was $21.9 \mathrm{ml} \times \mathrm{kg}^{-1} \times \mathrm{min}^{-1}$. Maximum ventilation was 55.2 litres per minute. Body fat determination via densiometric analysis yielded a figure or $36.9 \%$

\section{Discussions and conclusions}

It is known that when analysing human performance in any person who has a severe, permanent, physical disability the amount of remaining functional musculature is crucial (Bergh et al., 1976; Courts, Rhodes and McKenzie, 1983; Crews, Well, Burkett and Hopkins, 1982; Secher et al., 1974). This 25-year-old female athlete with paraplegia at approximately the T3/4 spinal level, had a measured $\dot{\mathrm{V}}_{\mathrm{O}_{2}}$ max of $21.9 \mathrm{ml} \times \mathrm{kg}^{-1} \times \mathrm{min}^{-1}$. Coutts et al. (1983), and Wicks, Oldridge, Cameron and Jones (1983), note maximum oxygen intake values in the range of 11.3 to $37.7 \dot{\mathrm{V}}_{\mathrm{O}_{2}} \mathrm{ml} \times \mathrm{kg}^{-1} \times \mathrm{min}^{-1}$. Maximum heart rates achieved in their subjects were too to 184 beats $\times \min ^{-1}$. These values for oxygen uptake ahd heart rates were derived mostly from studies of young male disabled subjects.

The important fact gleaned from these studies appears to be that the amount of functional musculature is related to work performance. Heart rates in Class $1 \mathrm{~A}$ individuals (quadriplegic) were low (approximately 100 beats $/ \mathrm{min}$ ), while the athlete who had more functional musculature (paraplegics) had heart rates of up to 184 beats per minute. Heart rate increases during work generally coincide with increases in maximal oxygen consumption (Åstrand and Rodahl, 1977; Lewis, Taylor, Graham, Pettinger, Schutte and Blomqvist, 1983; Morris, 1984). 
Another factor to consider is that sympathetic nervous drive to the heart is impaired in high level spinal cord injured people (Guttmann 1976).

The percent body fat reported in this ultramarathon competitor is slightly higher than the approximately $30^{\circ}$ o found in two elite female wheelchair athletes recently reported by Lussier, Knight, Bell, Lohman and Morris (1983). The level of the spinal injury appears to be a factor in the level of activity and hence, perhaps percent body fat (Morris, 1984, 1985).

Another consideration that should be noted in studying the disabled athlete is to look carefully at the different ergometers that may be used to test these individuals. In a recent paper, Moldover and Downey (1983) evaluated subjects using three different types of ergometers. They used an arm-crank ergometer, together with a cycle ergometer (subjects in sitting and supine position) in their six able-bodied, healthy male subjects. They concluded that myocardial oxygen requirements were different, and in fact, greater for the upright heavy exercise in the inclined (supine) position. Also, the arm-cranking exercise produced different responses when contrasted with lower extremity exercise in their subjects. Secher et al. (1974) have also indicated that different subjects (subjects with different amounts of muscle mass) display different maximal oxygen uptake capacities. In another recent study, Coutts et al. (1983) found greater maximum $\mathrm{O}_{2}$ uptake values in paraplegics than in tetraplegics.

It is important to relate maximal cardiac function, as well as respiratory performance, in people with different levels of spinal cord injury because in a recent U.S.A. National Championships there was a track event of 5 kilometres which was open to participants of all classes (paraplegics and tetraplegics). Also, many marathon races and road races are open to all wheelchair athletes regardless of their class (i.e. level of physical impairment).

These laboratory findings are important for two reasons: they provide physiological evidence that different class levels (paraplegics vs tetraplegics) should be employed in track, as well as in road racing events. This will allow more favourable competition and will allow more recognition of athletes who are disabled on many levels (Morris, 1985). Secondly, these data suggest that an athlete who trains by extending their long distances in practices and races can compete successfully in ultramarathon events (events at the marathon and 50-mile distances). This competitor, although possessing a severe, permanent, physical disability, was able to compete successfully alongside able-bodied competitors in endurance events by incorporating long distance training patterns in her preparation for the race. Endurance capacity was improved to the extent that this outstanding female athlete could maintain a racing pace of approximately $8 \mathrm{~min} / \mathrm{mile}$ for nearly seven hours in a 50 -mile race.

\section{References}

AKERS R, BUSKIRK ER 1969 An underwater weighing system utilizing 'force cube' transducers. Journal of Applied Physiology 26:649-653.

Ástrand P-O, Rodahl K 1977 Textbook of Work Physiology. McGraw-Hill, New York. Åstrand P-O, SALTin B 1961 Maximal oxygen uptake and heart rate in various types of muscular activity. Journal of Physiology 16:971-976.

Bergh U, Kanstrup IL, Ekblom B 1976 Maximal $\mathrm{O}_{2}$ uptake during exercise with various combinations of arm and leg work. Journal of Applied Physiology 41:191-196. 
Coutts KD, Rhodes EC, McKenzie DC 1983 Maximal exercise responses of tetraplegics and paraplegics. Journal of Applied Physiology: Respiratory, Environmental and Exercise Physiology 55:479-482.

Crews D, Well CL, Burkett L, Hopkins V 1982 A physiological profile of four wheelchair marathon racers. The Physician and Sportsmedicine 10:134-142.

Guttman L 1976 Textbook of Sport for the Disabled, HM \& M Publishers Ltd., Aylesbury, Bucks, England.

Lewis SF, Taylor WF, Graham RM, Pettinger WA, Schutte Je, Blomevist CG 1983 Cardiovascular responses to exercise as functions of absolute and relative work load. Journal of Applied Physiology: Respiratory, Environmental, and Exercise Physiology 54:1314-1323.

Lussier L, KNight J, Bell G, et al. 1983 Body composition comparison in two elite female wheelchair athletes. Paraplegia 21:16-22.

MAHLER DA, LoKe J 1985 The physiology of marathon running. The Physician and Sportsmedicine 13:85-97.

Moldover JR, Downey JA 1983 Cardiac responses to exercise: comparison of 3 ergometers. Archives of Physical Medicine and Rehabilitation 64:155-159.

MORRIS AF 1982 The track athlete needs medical classification. Sports 'n Spokes 7:3.

MorRIs AF 1983, April Wellness and Sports. Invited paper presented to national continuing education program, 'Recreation, Sports, and Wellness ... New Opportunities for the Disabled.' Rehabilitation Institute of Chicago, Chicago, Illinois.

MorRIs AF 1984 Sports Medicine: Prevention of Athletic Injuries, Wm. C. Brown, Dubuque, Iowa.

MORRIS AF 1985, in press An analysis of racing wheelchairs used at the 1980 Olympic Games for the Disabled. Research Quarterly for Exercise and Sport.

SECHER NH, RUBERg-LARSEN N, BinkHorst RA, et al. 1974 Maximal oxygen uptake during armcranking and combined arm plus leg exercise. 36:515-518.

Wicks JR, OldRIDGe NB, Cameron BJ, et al. 1983 Arm cranking and wheelchair ergometry in elite, spinal cord-injured athletes. Medicine and Science in Sports 15:223-231.

\section{Acknowledgements}

Appreciation is extended to Dr Richard Boileau (Director, Physical Fitness Research Laboratory) and his staff for help in the data collection portion of this study. 